\title{
HEMAGGLUTININ-ESTERASE GLYCOPROTEIN GENE OF BOVINE CORONAVIRUS DELIVERED BY ADENOVIRUS VECTOR INDUCES MUCOSAL IMMUNITY IN COTTON RATS
}

\author{
M. E. Baca-Estrada, L. A. Babiuk, and D. Yoo \\ Veterinary Infectious Disease Organization \\ University of Saskatchewan \\ Saskatoon, SK \\ Canada
}

Adenoviruses replicate in the mucosal surfaces of the respiratory and intestinal tracts of humans and animals. Therefore, recombinant adenoviruses are attractive live vectors for antigen delivery to induce effective mucosal immune responses. Enteric coronavirus is an important cause of neonatal diarrhoea in calves resulting in significant economic losses due to the mortality and decreased productivity of the survivors. The hemagglutinin-esterase (HE) of bovine coronavirus $(\mathrm{BCV})$ encodes a major viral membrane glycoprotein that elicits BCV-neutralizing antibodies (Deregt \& Babiuk, 1987). We constructed a recombinant adenovirus vector to carry the $\mathrm{HE}$ gene of $\mathrm{BCV}$ in the early transcription region 3 of the adenovirus genome (Yoo et al. 1992). Testing of recombinant human adenovirus in animals has been difficult due to the highly restricted host range. We have used cotton rats as a model to test the immunogenicity of our recombinant adenovirus vector, since they have been shown to support replication of various adenovirus including human Ad5 (Pacini et al 1984).

The aim of these study was to examine the ability of a recombinant adenovirus vector expressing the hemagglutinin-esterase glycoprotein of bovine coronavirus (AdBcHE) to induce mucosal and systemic immune responses following enteric immunization. Cotton rats were immunized intraduodenally with PBS, wild type (Ad) or recombinant adenovirus $(\mathrm{AdBcHE})$ and re-immunized orally with the same dose 21 days later. Our results demonstrated that enteric immunization with $\mathrm{AdBcHE}$ is capable of inducing significant levels of BCV-HE-specific IgG and IgA-secreting cells in the mesenteric lymph nodes (Table 1). In order to assess the ability of enteric immunization with AdBcHE to induce antibody responses in the respiratory tract, intraduodenally immunized cotton rats were inoculated intranasally with BCV. Only the animals immunized by the enteric route with 
Table 1. Antibody secreting cells in mesenteric lymph nodes and $B C V$ neutralizing titres in serum following intraduodenal immunization with AdBcHE

\begin{tabular}{lccccc}
\hline Immunization & Neut titre $^{\prime}$ & \multicolumn{2}{c}{ Anti-Ad $^{2}$ IgG IgA } & \multicolumn{2}{c}{ Anti-BCV IgG IgA } \\
\hline Ad & $<1$ & $23 \pm 7$ & $47 \pm 13$ & $5 \pm 4$ & $3 \pm 3$ \\
AdBcHE & 7.6 & $22 \pm 8$ & $52 \pm 12$ & $18 \pm 2$ & $23 \pm 9$ \\
\hline
\end{tabular}

' $\mathrm{BCV}$ neutralizing titres $\left(\log _{2}\right)$ in serum were determined by virus neutralization plaque assay. The endpoint was determined by the dilution which reduced plaque formation by $50 \% .{ }^{2}$ Antibody secreting cells (ASC) were measured by an ELISPOT assay and expressed as the mean \pm std of $\mathrm{ASC} / 10^{6}$ cells of 5 rats/group.

AdBcHE developed significant levels of BCV-HE-specific IgG and IgA antibodies in respiratory tract secretions measured by ELISA (Table 2). In addition, the serum, lung washes and nasal washes of $\mathrm{AdBcHE}$ immunized cotton rats contained antibodies capable of neutralizing BCV infectivity in vitro (Table 2).

We conclude, based on our results, that intraduodenal immunization of cotton rats with a recombinant human adenovirus expressing bovine coronavirus antigen HE induces systemic and mucosal antibody responses to Ad and BCV. In addition, enteric immunization of cotton rats with recombinant $A d B c H E$ vector primed cotton rats to develop significant $\mathrm{BCV}$-specific IgA responses in the respiratory tract following intranasal administration of BCV.

Our results demonstrate the potential of recombinant adenovirus for mucosal delivery of vaccine antigens and highlights their ability to prime distal mucosal immune responses where protective immunity may be beneficial.

\section{ACKNOWLEDGMENTS}

This work was supported by Health Services Utilization and Research Commission of Saskatchewan, the Natural Sciences and Engineering Research Council of Canada and the Medical Research Council of Canada. The authors thank Terry Beskorwayne and the staff of the animal care unit at VIDO for their expert technical assistance and Dr. Brian Underdown (McMaster University) for providing the anti-rat IgA antibody.

Table 2. Effect of intraduodenal (i.d.) immunization with AdBcHE on the immune response to intranasal (i.n.) administration of $\mathrm{BCV}$

\begin{tabular}{lccccc}
\hline & \multicolumn{2}{c}{ Immunization } & \multicolumn{3}{c}{ Anti-BCV } \\
Sample & i.d. & i.n. & Neut titre & IgA & Anti-BCVIgG \\
\cline { 2 - 4 } LW & Ad & BCV & $<1$ & $0.06 \pm 0.01$ & $0.09 \pm 0.01$ \\
& AdBcHE & BCV & 4.7 & $0.45 \pm 0.05$ & $0.46 \pm 0.07$ \\
NW & Ad & BCV & $<1$ & $0.13 \pm 0.04$ & ND \\
& AdBcHE & BCV & 6.3 & $0.45 \pm 0.05$ & ND \\
Serum & Ad & BCV & $<1$ & $0.002 \pm 0.003$ & $0.001 \pm 0.001$ \\
& AdBcHE & BCV & 7.6 & $0.01 \pm 0.01$ & $0.4 \pm 0.05$ \\
\hline
\end{tabular}

'See Table $1 .{ }^{2}$ Antibody responses against BCV were analyzed by ELISA in serum (at a dilution of $1 / 500$ ) and in lung washes (LW), nasal washes (NW) (at a dilution of 1/8). Not done (ND). Results are expressed as the mean \pm std of the optical density (O.D. $\left.{ }_{405}\right)$. 


\section{REFERENCES}

Deregt, D. and Babiuk, L. A. 1987. Monoclonal antibodies to bovine coronavirus: characteristics and topographical mapping of neutralizing epitopes on the E2 and E3 glycoproteins. Virology 161: 410.

Pacini, D. L., Dubovi, E. \& Clyde, W. A. Jr. 1984 A new animal model for human respiratory tract disease due to adenovirus. J. Infec. Dis. 150: 92.

Yoo, D., Graham, L., Prevec, L., Parker, M. D., Benko, M., Zamb, T. \& Babiuk, L. A. 1992 Synthesis and processing of the haemagglutinin-esterase glycoprotein of bovine coronavirus encoded in the E3 region of adenovirus. J. Gen. Virol. 73: 2591 